\title{
Decision uncertainties in natural hazard risk management: damage potential
}

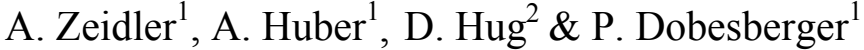 \\ ${ }^{1} B F W$ - Department of Natural Hazards, Innsbruck, Austria \\ ${ }^{2}$ Julius-Maximilians-University, Würzburg, Germany
}

\begin{abstract}
The infrastructure of energy production facilities is vulnerable to natural hazards, especially in Alpine environments. The determination of risk requires the knowledge of the probability of a natural hazard event and the associated intensity and the damage potential (vulnerability), which is site and object specific. Up to now a qualitative risk assessment was standard praxis in energy production facilities, but in the past years a quantitative risk analysis gained in importance, including the determination of uncertainties. In this paper we introduce a method on how to consider ranges of object values $(€)$ and ranges in the damage susceptibility of different types of objects in risk calculation.

Keywords: natural hazard, risk management, uncertainties, energy systems.
\end{abstract}

\section{Introduction}

In Austria, one sector vulnerable to natural hazards is the energy sector, where a sustainable supply strongly relies on an intact grid system. In the project RIMES (Climate Change and Risk Management in Energy Systems, co-funded by the ACRP) the main hypothesis states that climate change may influence natural hazard processes (avalanche, debris flow, permafrost) and subsequently lead to the need for improved risk management procedures. In most operations losses due to natural hazards are subordinated in the maintenance costs, except in the case of extreme events, making it difficult to determine the costs due to natural hazards and hindering an objective risk management procedure.

The focus of this paper lies on uncertainties associated with the determination of the damage potential in risk calculations. It comprises an overview of aspects in risk calculations, a description of the applied method to assess the damage 
potential of a hydropower generation facility and an example on how the factor damage susceptibility $(D S)$ in the determination of the damage potential (in some studies referred to as vulnerability) may contribute to uncertainties in decisions.

\section{Literature review}

\subsection{Uncertainties in natural hazard risk management}

Today it is widely accepted that uncertainties are inherent in any part of risk management and that decisions have to be made despite these uncertainties. Uncertainties may arise in data acquisition, data analysis, result communication and decision making [1]. Walker et al. [2] developed an uncertainty matrix, which includes the location of uncertainty (dataset, context and framing, input, parameter and model) and the level of uncertainty (statistical, scenario and recognized ignorance). The further distinction into the nature of uncertainty (aleatory and epistemic) is important as epistemic uncertainties can be reduced in a risk management process (e.g. [3]). Hug [1] (after [4]) describes these as:

- $\quad$ Epistemic uncertainty (Greek episteme = science) arises by subjective uncertainty, lack of knowledge, (limited) knowledge uncertainty, ignorance, imperfection, or specification error about the behavior of the system, which is related to our inability to understand, measure and describe.

- Aleatory uncertainty (lat. Alea = dice game) arises through natural, unpredictable variation in the performance of the system under study.

\subsection{Why does uncertainty analysis matter}

McEachren et al. [5] describe the uncertainty as a complex concept with many interpretations across knowledge domains and application contexts. However, uncertainty analysis is widely seen as a contribution to a better understanding of the existing systems and future developments. The costs for constructing mitigation measures are significantly lower at the time the objects at risk are build. However, due to the lack of comprehensive quantitative hazard and risk assessment methods it is difficult to perform a sustainable cost-benefit-analysis in the planning procedure. Consequently the management of natural hazard was dominated by reacting instead of preventing. Based on a review of existing literature Hug [1] (after: [6-8]) clustered key arguments into three classes:

1. Transparency - in analyzing, assessing and communicating risk. Allows a range in the outcome, which can be interpreted by the decision maker.

2. Improved knowledge about the process - by providing objective information on inherent uncertainties.

3. Minimize risk - by objectively comparing risks and subsequent management options and by providing additional information in the decision process. 
The arguments for uncertainty analysis seem strong; however, uncertainty analysis is still not standard procedure in most hazard assessments [6]. The reasons for the limited use of uncertainty analysis include aspects related to the complexity of natural hazard processes and the need for sophisticated statistical analysis that are time and cost intensive (e.g. [7, 9, 10]). In addition the end-user of the uncertain information may not know how to deal with these in the decisions to be taken. Some studies addressed this issue and introduced comprehensive concepts (e.g. [7]), but practical application is lacking behind.

As a summary Hug [1] states that "the crux of uncertainty analysis is to minimize the effort, but not to trade off important information. The effort is rising with increasing consideration of uncertainties, but does not guarantee a positive cost/benefit ratio". In addition the needs of the end-users have to be taken into account and one has to differentiate between a scientific study and the development of a practical tool.

\subsection{Frameworks to determine the damage potential}

Tacnet et al. [11] state that decisions have to be taken often based on imperfect information provided by more or less reliable sources and therefore highlights the importance of decision tools. This should help in handling and interpreting the results more reliable. In times of limited financial resources cost-benefit assessments for the evaluation of different mitigation options in risk management become increasingly important. In order to guarantee comparability and objectivity tools are provided by public authorities. In the last years several decision tools have been developed to help decision makers to effectively and efficiently deal with natural hazards. To this end the Swiss tool EconoMe [12] and the excel-based guidelines for cost-benefit assessments (in the following KNU) in Austria [13] have been developed that are supported by government (see [14]). Both frameworks introduce default values for the parameters in the damage potential equation $[3,5]$, which will be analyzed in the last part of this paper.

\section{Dealing with uncertainties in the assessment of the damage potential for a hydropower generation facility}

\subsection{Frame conditions}

The aim of RIMES is to develop a decision support tool for hydropower facilities to assess the risk due to natural hazards for today and for the future also considering climate change. By stating, visualizing and communicating uncertainties (e.g. as ranges in the outcome) sustainable decisions for the effective and efficient allocation of financial resources in mitigation measures shall be supported. This includes the presentation of the outcome in value ranges, instead of deterministic numbers, which reflects the possible range of outcome. In the project a participatory approach by involving the responsible decision makers of a hydropower facility was chosen to enable a goal oriented research 
environment. Due to time and manpower constraints the following boundary conditions apply:

1. Geographical: determination of risk for single hydropower generation facilities. The sum of risk of single objects leads to the collective risk of the facility.

2. Conditional: climate and climate change and the subsequent determination of hazard scenarios are based on precipitation and temperature for a planning horizon of 40 years; the natural hazard processes are avalanches, debris flows and sedimentation processes due to melting of permafrost.

3. Contextual: economical damages: direct (damages on infrastructure of hydropower facility) and indirect (losses due to the disruption of the energy production); costs are based on today's values.

Keeping to the frame conditions we acknowledge that important aspects such as ecological and social damages, other natural hazard processes, changes in vegetation, land use changes and a possible higher capacity of the production facility are neglected.

\subsection{Calculating risk}

The concept of risk is commonly described as a function of probability and consequences and can be expressed as (after [15]):

$$
R=p * C
$$

where $R$ is the risk, $p$ the probability of occurrence and $C$ the consequences.

Equation (1) is broadly formulated and comprises many aspects of risk considerations. In any step of a risk management procedure it has to be clearly stated what kind of data and model was used in the specific study. More specific problems usually require more detailed information, e.g. on a regional level a more general index for the damage potential (average value for houses) maybe sufficient, whereas for the planning of a local mitigation measure the value of the object at risks needs to be more accurate. This is adding more sources of uncertainty to the parameter value of object, because more data are needed for the determination of the value. In the present study we determine the collective risk for energy production facilities on an object basis, which requires a detailed analysis on the damage potential.

For the risk calculation in this study the Equations (1) and (2) of the Swiss guidelines are used without analyzing the uncertainties inherent to the models itself.

\subsection{Determining the damage potential}

As outlined above, for any cost-benefit analysis it is important to reliably assess the damage potential of each object at risk. The consequences $(C)$ in Equation (1) are the damages caused by natural hazard event impacts on objects of the 
hydropower facility $(D(O))$. After Bründl and Romang [16] the damage can be calculated as:

$$
D(O)_{i, j}=\left(1-\varepsilon_{i}\right) * V(O)_{i} * D S(O)_{i, j}
$$

where $D(O)_{i, j}$ is the potential damage $(€)$ to an object $\left(O_{i}\right)$ caused by a scenario $\left(S_{j}\right) . \varepsilon_{i}$ is a factor accounting for structural mitigation measures. $V(O)_{i}$ is the value $(€)$ of the object at risk and $D S(O)_{i, j}$ reflects the damage susceptibility of the object $\left(O_{i}\right)$ to the given scenario $\left(S_{j}\right)$.

$D(O)_{i, j}$ depends on the type and intensity of natural hazard processes.

As is implied in Equation (2), the damage potential is a function of the value of an object, the damage susceptibility $(D S)$ of an object in relation to the intensity of a process and a factor introduced to account for mitigation measures. The uncertainties in these parameters arise from imperfect information, but the severity on decisions has to be assessed for each specific case. Often site-specific information are neglected (e.g. construction types, terrain factors), which may be reflected in too high or too low values of the $D S$. Additionally, there is not sufficient data to quantify the extent of the damage of individual objects depending on the impact pressure. Consequently, there is uncertainty in the input data for the calculation of the damage potential. The uncertainty is epistemic as it can be reduced in the case that more reliable information becomes available.

\subsubsection{Selecting objects at risk and scenarios}

Before starting to calculate the damage potential relevant objects and scenarios have to be identified to meet the aims of the project. The following object classes were selected by representatives of the hydropower facility for which a risk calculation should be performed:

1. Access roads and trails

2. River catchment intakes and diversions

3. Above ground powerlines (valley and hill site) including towers

4. Energy production buildings

5. Outdoor substations

6. Blockages/damms

7. Reservoirs

Based on a questionnaire one or more objects at risk were identified for each object class by local experts. In total app. 60 single objects are considered.

Having identified all relevant objects at risk, for each of these objects likely hazard scenarios were determined as depicted in Figure 1 on the example of avalanche hazard on electricity towers. Hence, five hazard scenarios are considered in the determination of the damage potential. Within each of these scenarios the intensities of the process is assessed for five return periods (5-, 30-, $70-, 100$ - and 300-years). 


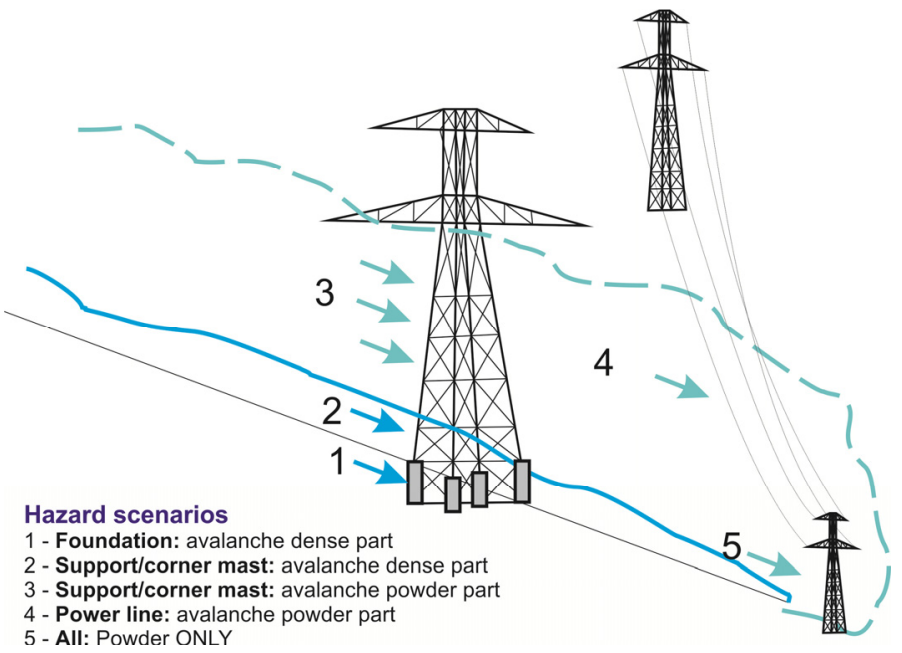

Figure 1: Avalanche hazard scenarios for electricity tower.

Now, that the hazard scenarios and the associated intensities are estimated these have to be related to the expected extent of damage.

Under the assumption that the objects at risk and the intensity of the process scenarios are given it is necessary to relate the expected damage to the intensity. This is quite difficult as only few measured impact pressures on objects are available. One of the few studies in this regard was carried out by Fuchs et al. [17] who empirically analyzed the vulnerability of buildings to debris flows and established a vulnerability function. Generally the extent of damage depends on the process and on specifications of the object. (e.g. [18]) such as:

- $\quad$ Type of process (e.g. power or dense flow avalanche, debris flow)

- The height of the impact

- The angle of the impact

- $\quad$ Type of design of object

- The basis of assessment for building the object

- $\quad$ Existing measures to protect the object (e.g. elevated foundation)

Considering single objects at risk, the representatives of the hydropower facility stated that it would be interesting for their decision to group the damages into four classes (small, medium, large, catastrophic damages) in regard to their degree of damage to a specific object rather than to the total amount of costs. In Figure 2 the damage scenarios considered in RIMES are shown. A damage is considered small, if the cost for repairing the object does not exceed $2 \%$ of the specific cost; a catastrophic damage occurs when at least $50 \%$ of the building costs arise. Consequently, the $D S$ in Equation (2) is directly related to the limits of the damage classes: 0-0.02 (small), 0.02-0.1 (medium), 0.1-0.5 (large) and 0, 5-1 (catastrophic). 


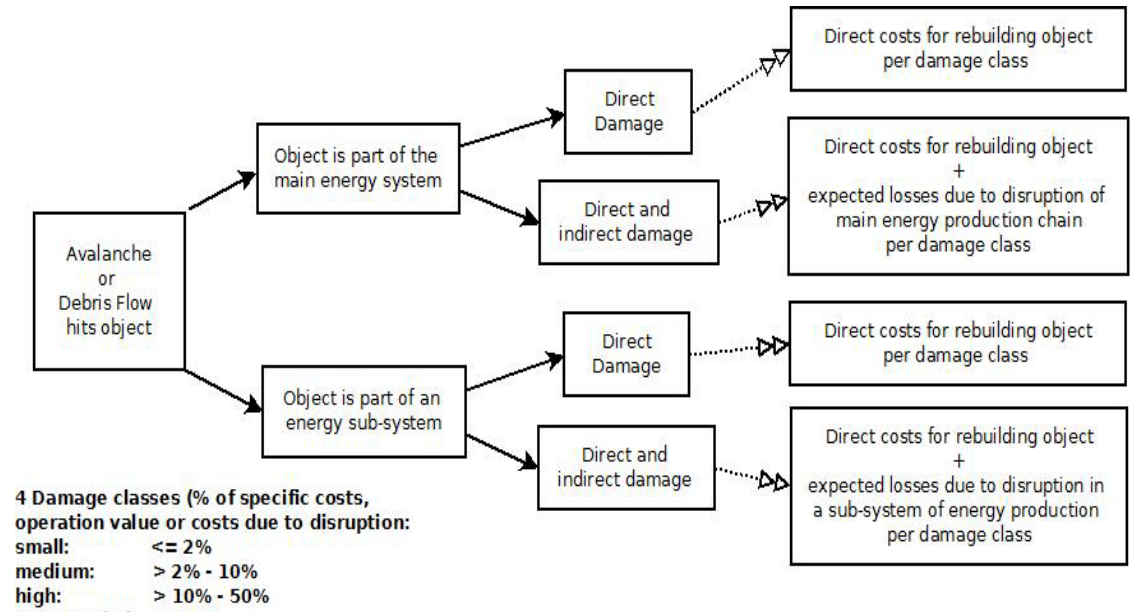

Figure 2: Damages considered in RIMES.

\subsection{Calculating the damage potential $D(O)_{i, j}$ on the example of an electricity tower}

\subsubsection{Assumptions in this example}

The following assumptions are made in this paper in order to assess the uncertainty in the damage susceptibility $(D S)$ :

\section{Object at risk $(\boldsymbol{O})$ : $\quad$ Electricity tower}

\section{Hazard scenario: $\quad$ 1. Dense flow avalanche: $7 \mathrm{kPa}$ impact pressure}

2. Dense flow avalanche: $35 \mathrm{kPa}$ impact pressure

Probability of event: 30 years

$$
\boldsymbol{V}(\boldsymbol{O})_{\text {electricity tower }}: \quad 120.000-200.000 € \text { (average } 160.000 € \text { ) }
$$

$\varepsilon$ :

0 , meaning that no measure is applied to protect the electricity tower

Equation (3) shows that the only missing parameter is $D S$, which will be investigated in Section 3.4.2.

$$
D(O)_{i, j}=(1-0) * V(O)_{i} * D S(O)_{i, j}
$$

For $\mathrm{i}=$ electricity tower, $\mathrm{V}(\mathrm{O})_{\mathrm{i}} \approx 120.000-200.000 €$

\subsubsection{Damage susceptibility (DS)}

Please note that the pressure values in RIMES are assumptions in the present paper as here the intention is only to highlight the uncertainties in calculating the damage potential using $D S$. The determination of the intensity leading to the damage classes is part of a subsequent paper. Anyhow, in the following the range 
of possible $D S$ in the damage classes and the range of costs for electricity towers are considered using a Monte Carlo Simulation.

In this paper an improvement in the risk calculation of a hydropower facility is anticipated by showing the ranges of outcome using a Monte Carlo simulation for $D S$ in the four damage classes as outlined above and the range of object values (120.000-200.000€). As a comparison the standard values for $D S$ in the Austrian KNU and the Swiss EconoMe [19] tools are included in the analysis. Both tools do not allow for ranges in object values and therefore the average value $(160.000 €)$ is used in the calculation for comparison. As mentioned above both decision tools, KNU and EconoMe, suggest default values for the damage sensitivity $(D S)$. They are based on the intensities in the respective hazard zone mapping regulations and for avalanches the intensity-damage relations for electricity towers are as follows (see also Figure 3):

KNU: $\quad<10 \mathrm{kPa}($ yellow zone $)-\boldsymbol{D S}=0,5 ;>10 \mathrm{kPa}($ red zone $)-\boldsymbol{D S}=1$

EconoMe: $<3 \mathrm{kPa}$ (low intensity) $-\boldsymbol{D S}=0,005 ; 3-30 \mathrm{kPa}$ (medium intensity) $\boldsymbol{D S}=0,3 ;>30 \mathrm{kPa}$ (high intensity) $-\boldsymbol{D S}=1$

RIMES: $\quad<5 \mathrm{kPa}-\boldsymbol{D S}=0-0,02 ; 5-15 \mathrm{kPa}-\boldsymbol{D S}=0,02-0,1 ; 15-30 \mathrm{kPa}-$ $\boldsymbol{D S}=0,1-0,5 ;>30 \mathrm{kPa}-\boldsymbol{D S}=0,5-1$

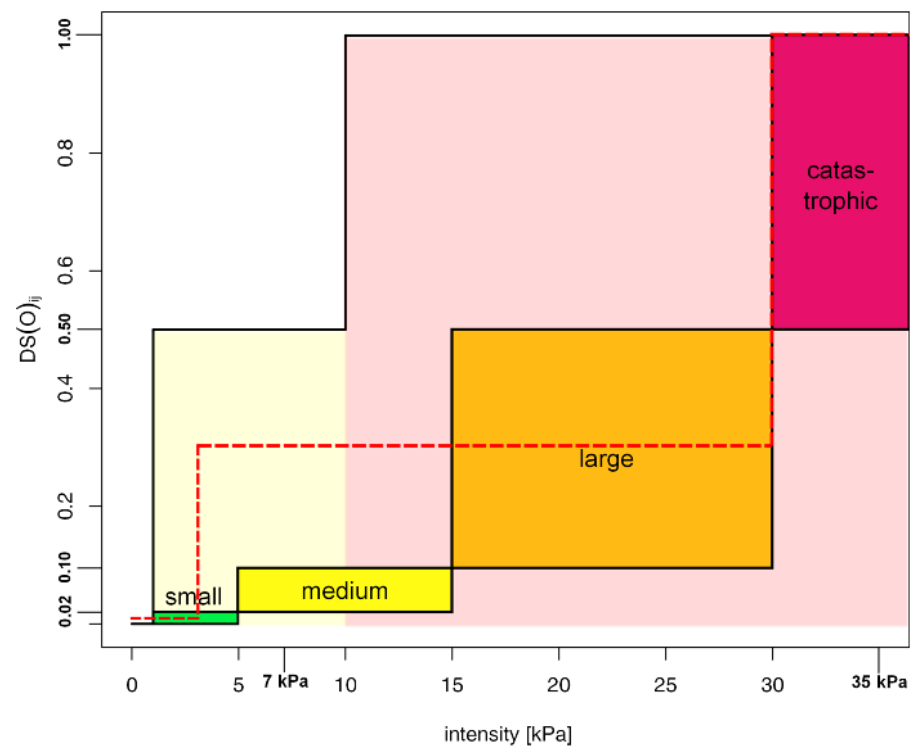

Figure 3: Intensity-damage relations. The colored boxes indicate the relations as used in the present study. The solid black line represents the relation proposed by the KNU. The dashes red line represents EconoMe data. The boxes in the background represent the red and yellow hazard zone for avalanches (Austria). 


\subsubsection{Results}

The following example illustrates the application of the methodology. Figure 4 shows the intensity-relation, with the respective $D S$ for an intensity of $7 \mathrm{kPa}$ and $35 \mathrm{kPa}$. With the proposed method in a first step the corresponding damage class for the given intensity is selected. In a second step the damage susceptibility $D S(O)$ and the $V(O)$ are treated as random variables and a probability distribution is selected based on the chosen damage class. For the given examples a normal distribution was chosen to represent the random variable with a standard deviation of $0,02(7 \mathrm{kPa})$ and $0.125(35 \mathrm{kPa})$. In the next step a Monte Carlo Simulation is performed in order to obtain a set of values for $D(O)$. The results of the procedure are presented in Figures 5 and 6 . Results obtained by application of the vulnerability functions after KNU and EconoMe are presented for comparison.

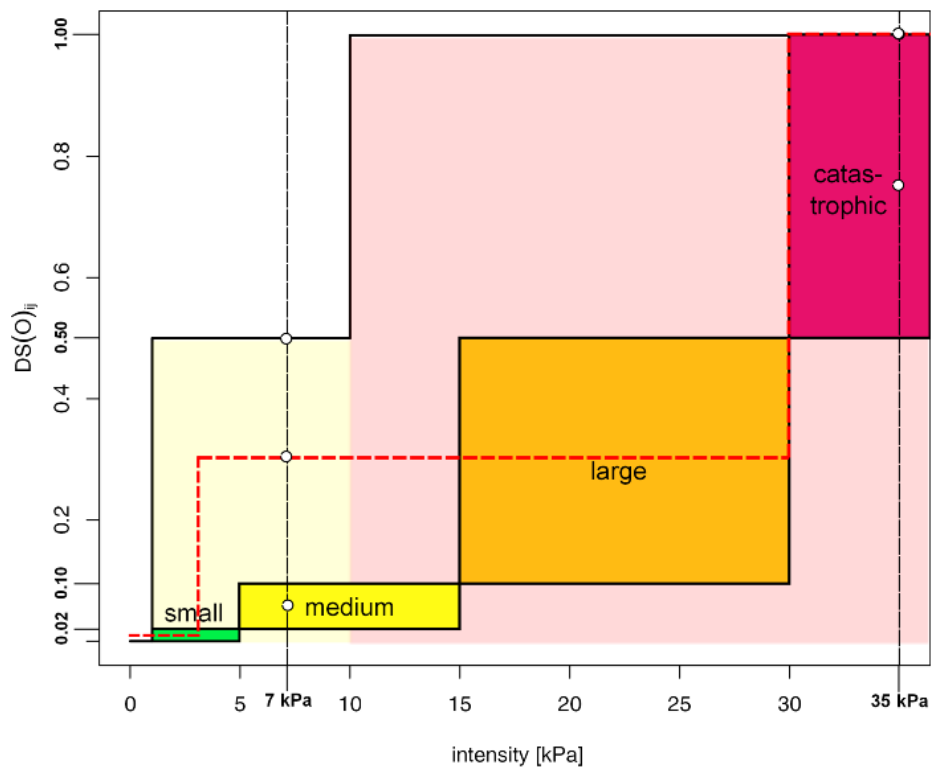

Figure 4: Example of an event with given intensity of $7 \mathrm{kPa}$ and $35 \mathrm{kPa}$. The white dots depict the respective vulnerability of the object $D S\left(O_{i, j}\right.$ to the respective event.

The results in Figure 5 and 6 show the range of outcome for the chosen example in this study. For the $7 \mathrm{kPa}$ scenario the damage potential lies between $2715 €$ and $16450 €$ with an average of $9585 €$ resulting in yearly risks (under the assumption of a 30 year event) of $91-548 €$ with an average of $320 €$, whereas using the standard value of the KNU the yearly risk is $2667 €$ (damage potential $80000 €$ ) and in EconoMe $1600 €$ (damage potential $48000 €$ ).

For the $35 \mathrm{kPa}$ scenario the damage potential lies between $68050 €$ and $171500 €$ with an average of $119750 €$ resulting in yearly risks of $2268-5717 €$ 


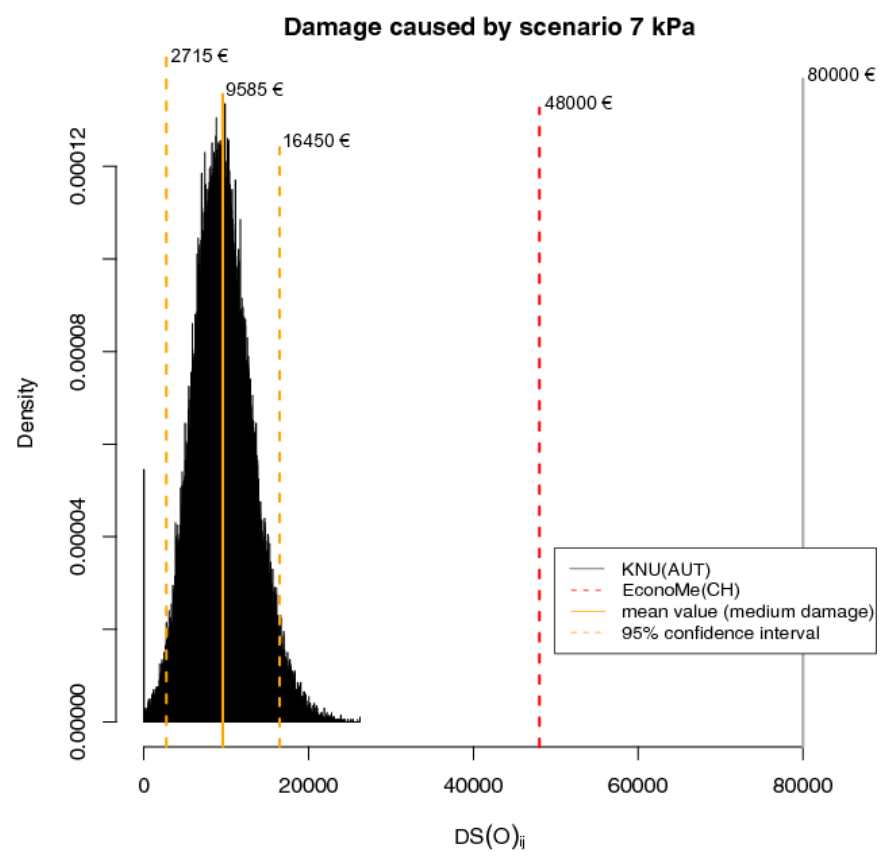

Figure 5: $\quad$ Results of the $7 \mathrm{kPa}$ scenario.

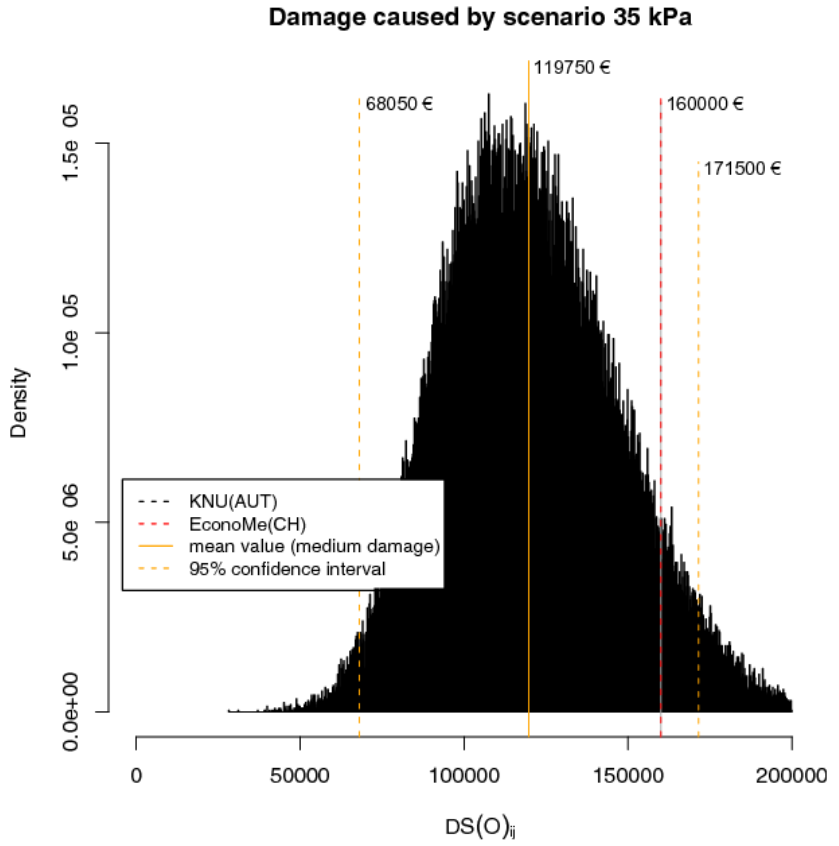

Figure 6: Results of the $35 \mathrm{kPa}$ scenario. 
with an average of $3992 €$, whereas using the standard value for both the KNU and EconoMe the yearly risk is $5333 €$. Because in the calculation the average value of the range of costs for the electricity towers was used the maximum range of yearly risk in the Monte Carlo Simulation is higher than the yearly risk using KNU and EconoMe.

\section{Conclusion}

The introduced approach to treat the damage susceptibility factor $D S$ in the specific case of the economical decisions of a hydropower facility in regard to the natural hazard management shows that a careful selection of the $D S$ is important, because this factor highly influences the outcome in risk calculations.

The differentiation into four damages classes, instead of using the suggested intensities in the KNU and EconoMe, is intended to account for different construction types of objects. Anyhow the intensities still have to be assessed. In the lower damage classes this representation of costs shall allow a more efficient planning of mitigation measures and help in the selection of new sites for planned objects.

\section{Acknowledgements}

We would like to thank all the employees of the VERBUND Hydro Power AG for the valuable discussions and for support in data acquisition. Also thanks to Peter Gauer and Karl Kleemayr for proof reading and discussions.

This project is funded by the Climate and Energy Funds within the framework of the ACRP (Austrian Climate Research Programme).

\section{References}

[1] Hug, D., Uncertainties in natural hazard risk management, Master Thesis, Julius-Maximilians-Universität Würzburg, 2012.

[2] Walker, W.E., Harremoës, P., Rotmans, J., van der Sluijs, J.P., van Asselt, M.B.A., Janssen, P. and Krayer von Krauss, M.P., Defining uncertainties. Integrated Assessment, 4(1), pp. 5-17, 2003.

[3] Merz, B. and Thieken, A.H., Flood risk curves and uncertainty bounds. Nat Hazards, 51, pp. 437-458, 2009.

[4] Kunz, M., Grêt-Regamey, A. and Hurni, L., Visualization of uncertainty in natural hazards assessments using an interactive cartographic information system. Nat Hazards, 2011.

[5] McEachren, A. M., Robinson, A., Hopper, S., Gardner, S., Murray, R. and Gahegan, M., Visualizing Geospatial Information Uncertainty: What we know and what we need to know. Cartographic and Geographic Information Science, 32(3), pp. 139-160, 2005.

[6] Pappenberger, F. and Beven, K.J., Ignorance is bliss: or seven reasons not to use uncertainty analysis. Water Resources Research, 42, 8 pp, 2006. 
[7] ASTRA Bundesamt für Straßen, Methodik zur vergleichenden Risikobeurteilung, 148 pp, 2009.

[8] Katz, R.W., Techniques for estimating uncertainty in climate change scenarios and impact studies. Climate Research, 20, pp. 167185, 2002.

[9] Paté-Cornell, M.E., Uncertainties in risk analysis: Six levels of treatment. Reliability Engineering and System Safety, 54(2-3), pp. 95-111, 1996.

[10] Peterson, S., Uncertainty and economic analysis of climate change: A survey of approaches and findings. Environmental Modeling and Assessment, 11, pp. 1-17, 2006.

[11] Tacnet, J.M., Batton-Hubert, M., Dezert, J. and Richard, D., Decision support tools for natural hazards management under uncertainty. Proc. of the $12^{\text {th }}$ Congress of Interpraevent, eds. G. Koboltschnig, J. Hübl and J. Braun, France, pp. 597-608, 2012.

[12] BAFU Bundesamt für Umwelt. Über EconoMe. http://www.econome. admin.ch/doku start.php

[13] BMLFUW Bundesministerium für Land- und Forstwirtschaft, Umwelt und Wasserwirtschaft, Richtlinien für die Wirtschaftlichkeitsuntersuchung und Priorisierung von Maßnahmen der Wildbach- und Lawinenverbauung, Wien, 53 pp, 2006.

[14] Bründl, M., Romang, H.E., Bischof, N. and Rheinberger, C.M., The risk concept and its application in natural hazard risk management in Switzerland. Natural Hazards Earth Systems Science, 9, pp. 801-813, 2009.

[15] Varnes, D.J. and the International Association of Engineering Geology Commission on Landslides and Other Mass Movements, Landslide hazard zonation: A review of principles and practice. Natural Hazards, (3), UNESCO, 63 pp. 1984.

[16] Bründl, M. and Romang, H.E., Teil A: Allgemeine Darstellung des Risikokonzepts. PLANAT, pp. 61, 2009.

[17] Fuchs, S.; Heiss, K. and Hübl, J., Towards an empirical vulnerability function for use in debris flow risk assessment. Natural Hazards and Earth System Sciences, 7 (5), pp. 495-506, 2007.

[18] Suda, J. and Rudolf-Miklau, F., Bauen und Naturgefahren. Springer Verlag: Wien and New York, 510 pp. 2012.

[19] Bründl, M. and Romang, H.E., Teil B: Anwendung des Risikokonzepts: Prozess Lawine. PLANAT, 38 pp. 2009. 